\title{
¿Pueden los ácidos grasos omega 3 y 6 contrarrestar los efectos negativos de la obesidad en la gestación?
}

\author{
Johana Escobar H. ${ }^{a}$, Lina Estrada A. ${ }^{\text {, Lina Gómez G. }}{ }^{a}$, Aura María Gil V., MSc ${ }^{b}$, \\ Angela Cadavid J., PhDC.
}

Grupo Reproducción, Sede de Investigación Universitaria (SIU). Universidad de Antioquia. Medellín, Colombia.

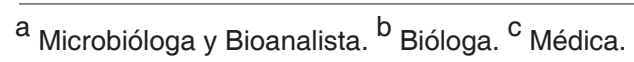

\section{RESUMEN}

La obesidad se define como el exceso de grasa corporal que pone en peligro la salud, aumentando la morbilidad y la mortalidad de la población. El índice de masa corporal es el indicador más usado para la determinación de la obesidad, y durante el embarazo se calcula de acuerdo a las modificaciones de RossoMardones. Según la Organización Mundial de la Salud, la prevalencia de la obesidad en el embarazo oscila desde 1,8 hasta 25,3\%, y la obesidad antes y durante la gestación constituye un importante factor de riesgo tanto para las complicaciones maternas como fetales. En la presente revisión de literatura, se detallan los efectos desencadenados por la obesidad gestacional, como los cambios en el perfil lipídico y el aumento significativo de la respuesta inflamatoria y del estrés oxidativo. A su vez, se describe la función de los ácidos grasos omega en el embarazo, los cuales han mostrado disminuir los efectos nocivos en las mujeres gestantes obesas, debido a su actividad hipolipemiante, antiinflamatoria y antioxidante.

\section{PALABRAS CLAVE: Obesidad, embarazo, hiperlipidemia, inflamación, estrés oxidativo, ácidos grasos omega}

\section{SUMMARY}

Obesity is defined as an excess of body fat that endangers the health, increasing morbidity and mortality of the population. The body mass index is the indicator used to determine obesity, and during pregnancy is calculated according to changes in Rosso-Mardones. According to the World Health Organization, the prevalence of obesity in pregnancy ranges from 1.8 to $25.3 \%$, and obesity before and during pregnancy is an important risk factor for both maternal and fetal complications. In this review of literature detailing the effects triggered by gestational obesity, such as changes in lipid profile, and significant increase of the inflammatory response and oxidative stress. In turn, it describes the role of omega fatty acids in pregnancy, which has shown to decrease the harmful effects in obese pregnant women because of their lipid lowering, antiinflammatory and antioxidant activities.

KEY WORDS: Obesity, pregnancy, hyperlipidemia, inflammation, oxidative stress, omega fatty acids 


\section{INTRODUCCIÓN}

Durante el embarazo se producen diversos cambios fisiológicos y metabólicos, que dan lugar a la formación y/o transformación de estructuras maternas necesarias para la gestación, como la placenta y las glándulas mamarias. A su vez, estos cambios, propician el aumento de depósitos de grasa requeridos para el crecimiento y el desarrollo fetal (1); sin embargo, cuando se presenta exceso de grasa, puede desencadenarse la obesidad gestacional y aumentar la probabilidad de complicaciones en el parto y enfermedades en el embarazo como la diabetes y la preeclampsia (2).

Es importante que la mujer tenga un peso adecuado antes y durante el embarazo. En primera instancia, se debe considerar el peso preconcepcional de la madre, el cual se clasifica según Rosso-Mardones por el Índice de Masa Corporal (IMC): peso bajo $\leq 19,8$, peso normal 19,8 a $<26,1$; sobrepeso 26,1 a $<29$ y obesidad $\geq 29$. Durante la gestación, es normal que la madre aumente de 11 a $16 \mathrm{~kg}$ de peso que favorecen el desarrollo fetal, distribuidos así: 1,5 a $1,8 \mathrm{~kg}$ en el primer trimestre; $3,5 \mathrm{~kg}$ en el segundo y el resto en el tercero $(0,4 \mathrm{~kg} /$ semana) (3). De igual manera, los diferentes componentes del perfil lipídico aumentan, originando un estado de estrés oxidativo, que no es perjudicial para la madre ni el feto; sin embargo, en las mujeres gestantes con obesidad, estos dos eventos se presentan de una forma exacerbada, conllevando a estados inflamatorios y oxidativos en la madre que alteran el transcurso normal del embarazo (4).

Los ácidos grasos omega han mostrado contrarrestar las complicaciones generadas por la obesidad debido a su actividad hipolipemiante, antiinflamatoria y antioxidante (5). Por este motivo, el objetivo de la presente revisión de literatura es resaltar el papel que podría tener la dieta rica en ácidos grasos omega en las mujeres gestantes con obesidad.

\section{HIPERLIPIDEMIA}

Generalidades. La hiperlipidemia es un trastorno caracterizado por la elevación anormal del colesterol (CT) y/o triglicéridos (TG) en el plasma, que puede presentarse debido a alteraciones en el proceso de absorción de las grasas de la dieta, en la síntesis endógena de lípidos y en el transporte reverso de CT al hígado. EI CT se puede encontrar libre o esterificado con ácidos grasos, y ambas formas, al igual que los TG y los fosfolípidos, circulan en la sangre unidas a proteínas específicas denominadas apolipoproteínas, formando complejos macromoleculares denominados lipoproteínas (6).
Metabolismo de los lípidos durante el embarazo. Durante el embarazo, la unidad feto placentaria se desarrolla a expensas de la madre, presentándose dos etapas: 1) etapa anabólica, que corresponde a los dos primeros tercios del embarazo y es cuando se incrementan los depósitos grasos de la madre; 2) etapa catabólica, se da lugar en el último tercio del embarazo, y es cuando se produce una movilización acelerada de las reservas grasas de la madre hacia el feto (7) (Figura 1).

El metabolismo lipídico durante el primer trimestre del embarazo se caracteriza porque las lipoproteínas de alta densidad (HDL) unidas al CT (HDLC), y el CT libre están ligeramente aumentados; por el contrario, las lipoproteínas de baja densidad (LDL) unidas al CT (LDL-C), los TG y las lipoproteínas de muy baja densidad (VLDL) disminuyen en comparación con las mujeres no embarazadas, y con el segundo y tercer trimestre de la gestación (8). En el segundo trimestre del embarazo, el perfil lipídico aumenta significativamente y en el tercer trimestre, la concentración de CT, LDL-C, HDL-C, TG y VLDL incrementa en comparación con las mujeres no gestantes. Por su parte, las HDL alcanzan su máxima concentración en la semana 25 , decrece en la semana 32 y a partir de ésta, se mantiene constante el resto del embarazo, y las LDL alcanzan su máxima concentración cerca de la semana 36 , como consecuencia de los efectos hepáticos del estradiol y de la progesterona (8). De otro lado, el transporte de TG no se reduce durante la gestación pero éste se ve afectado cuando las concentraciones de CT son altas o con la disminución de la lipoproteína lipasa encargada de hidrolizar a los TG de las LDL, descomponiéndolos en ácidos grasos y glicerol, lo que conlleva a un estado de hiperlipidemia en la madre durante toda la gestación (8). Las mujeres con obesidad en el embarazo presentan una alteración significativa en el perfil lipídico, comparado con el que presentan las mujeres gestantes con IMC inferior a $30 \mathrm{~kg} / \mathrm{m} 2$ (9).

En conclusión, durante la gestación es usual que aumenten los componentes del perfil lipídico, los cuales no son perjudiciales para la madre y el feto; sin embargo en las mujeres gestantes con obesidad, este aumento se produce de manera exacerbada, lo que conlleva a alteraciones metabólicas en la madre y finalmente, puede afectar el desarrollo normal del embarazo y del feto. Específicamente, cuando se presenta un desorden lipídico durante la gestación, la madre tiene un mayor riesgo de presentar diabetes mellitus, síndrome nefrótico, partos prematuros, alteración en el desarrollo de la placenta y preeclampsia. En el feto por su parte, este desorden lipídico puede causar lesiones en los vasos sanguíneos con aumento de la morbimortalidad (4). 


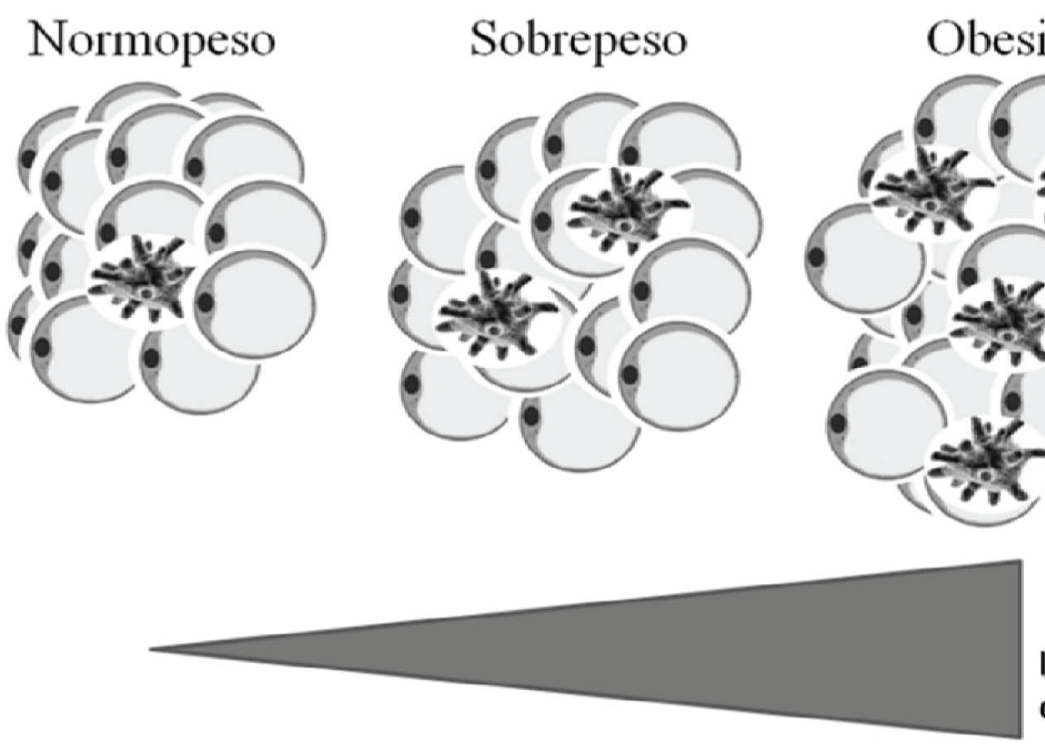

\section{Adipocitoquinas}

Perfil lipídico

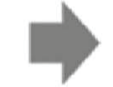

Activación

\section{Inflamación Oxidación}

\section{Inhibición}

\section{Aumento de la \\ concentración de lípidos plasmáticosy del almacenamiento de grasa}

\section{Acidos grasos omega-3 y omega-6}

Figura 1. Adaptación metabólica durante la gestación. En condiciones normales, la gestación requiere de una adaptación metabólica especial para la remodelación del tejido adiposo. Si bien, es necesario un aumento de la concentración de lípidos plasmáticos y del almacenamiento de grasa, a medida que aumenta el índice de masa corporal de la gestante, este proceso se realiza de una manera exacerbada, incrementando la secreción de adipocitocinas y del perfil lipídico. Lo anterior, conlleva a un aumento en la expresión de genes proinflamatorios que produce un estado de inflamación, el cual es contrastado por la actividad antinflamatoria de los omegas-3 y omega-6.

\section{INFLAMACIÓN EN LA OBESIDAD}

El tejido adiposo ha sido tradicionalmente considerado como un órgano de almacenamiento de energía. Las células propias de este tejido son los adipocitos, los cuales tienen la capacidad de secretar adipocitocinas, y sustancias vasoactivas (10). Las adipocitocinas se dividen en dos grupos: 1) moléculas específicas del tejido adiposo como la adiponectina, la leptina, la resistina y la visfatina y, 2) moléculas no específicas secretadas por otros tejidos además del tejido adiposo, como son el TNF, la interleuquina 1 (IL-1), la interleuquina 6 (IL6), el inhibidor del activador del plasminógeno tipo 1 (PAI-1) y la angiotensina (10). Se ha encontrado una correlación positiva entre las adipocitocinas y el IMC, la circunferencia de la cintura, la trigliceridemia, la insulinemia y la resistencia a la insulina; además, estas citocinas se encuentran implicadas en la regulación del peso corporal (leptina, adiponectina), la activación del sistema inmune (TNF, IL-1, IL-6, resistina) y la función vascular (angiotensina, PAI-1) (11).

En las mujeres gestantes hay diferencias en la concentración de adipocitocinas con respecto a las mujeres no gestantes, ya que algunas de estas moléculas, específicas del tejido adiposo como la resistina y la leptina son producidas por la placenta (11). En la Tabla I se presenta un resumen de cómo se regula la concentración de adipocitocinas durante la gestación normal. 
Tabla I

REGULACIÓN DE ADIPOCITOCINAS DURANTE LA GESTACIÓN NORMAL

\begin{tabular}{lllll}
\hline & NO GESTACIÓN & & GESTACIÓN & \\
& & Primer trimestre & Segundo trimestre & Tercer trimestre \\
\hline RESISTINA & Concentración basal & Concentración basal & Concentración basal & Incremento \\
LEPTINA & Concentración basal & Incremento & Incremento & Incremento \\
ADIPONECTINA & Concentración basal & Incremento & Disminución & Disminución \\
VISFATINA & Concentración basal & Concentración basal & Incremento & Disminución \\
\hline
\end{tabular}

Resistina. El papel de la resistina en la madre se ha relacionado con un estado de sensibilidad reducida a la insulina en las últimas etapas del embarazo, contribuyendo a la presentación de la hiperglicemia postprandial, la cual favorece el desarrollo fetal (11). Adicionalmente, Di Simone y cols (12), encontraron que la resistina es capaz de incrementar la capacidad invasiva de líneas trofoblásticas de primer trimestre y, estudios más recientes, han demostrado el papel positivo de esta adipocitocina sobre el proceso de angiogénesis, en donde la resistina estimuló la proliferación, migración y formación de estructuras tubulares por células endoteliales.

Las concentraciones séricas de resistina son similares en mujeres no gestantes y mujeres gestantes que se encuentran en el primer o segundo trimestre de la gestación; sin embargo, esta adipocitocina incrementa significativamente durante el tercer trimestre del embarazo (13). La expresión de resistina placentaria es significativamente mayor en la placenta a término que en las vellosidades coriónicas de primer trimestre, confirmando que la producción placentaria de ésta, representa la principal causa del incremento de resistina sérica durante la gestación (13).

Leptina. La placenta humana expresa altas cantidades de ARN mensajero y proteína de la leptina durante todo el embarazo (11), sin embargo, incrementa significativamente en el primer y en el último trimestre de la gestación comparado con mujeres no gestantes, y disminuye a concentraciones pregestacionales inmediatamente después del parto. La producción de leptina y de los receptores de leptina de las variantes $\mathrm{OB}-\mathrm{Rb}$ y $\mathrm{OB}-\mathrm{Ra}$ se encuentran localizados en las células del sincitiotrofoblasto que están en contacto con la sangre materna, con el citotrofoblasto extravelloso y con el endotelio vascular fetal. Por su parte, se ha sugerido que la leptina promueve la invasión trofoblástica y tiene un papel importante en el proceso de la angiogénesis, posiblemente al estar involucrada en la formación de nuevos vasos placentarios (11). De otro lado, las concentraciones de leptina están incrementadas en embarazos asociados con preeclampsia, IMC >25 $\mathrm{kg} / \mathrm{m} 2$, diabetes gestacional 0 hiperinsulinemia (14).

Adiponectina. La función de la adiponectina es mejorar la sensibilidad a la insulina, actuando a través de receptores específicos en el músculo esquelético y en el hígado, en donde es capaz de incrementar el consumo de glucosa e inhibir la gluconeogénesis, respectivamente (11). Sin embargo, esta acción podría prevenir que se produjese un estado diabetogénico fisiológico entre el segundo y el tercer trimestre del embarazo, absolutamente requerido para el rápido crecimiento fetal. Además, se ha observado que la adiponectina suprime el transporte transplacentario de aminoácidos y nutrientes mediado por insulina; por lo tanto, se ha sugerido que durante el embarazo disminuyen las concentraciones de adiponectina que contribuyen a la pérdida de sensibilidad a la insulina y que esta reducción se produce principalmente entre el segundo y el tercer trimestre del embarazo. Sin embargo, se han encontrado mayores concentraciones de adiponectina en la gestación comparado con las encontradas en el período posparto (11). La adiponectina es capaz de modular el proceso de la placentación al favorecer la migración y la diferenciación trofoblástica sin necesidad de inducir e incrementar la respuesta mitogénica. Adicionalmente, se ha considerado que la adipocitocina tiene un efecto inmunomodulador en la interfase materno-fetal debido a su capacidad de incrementar la expresión de CD24 y Siglec10 en células trofoblásticas a término. Ambas moléculas tienen la capacidad de regular negativamente la respuesta inmune materna ante los antígenos fetales (15). De otro lado, se ha observado que la adiponectina propicia in vivo la diferenciación de las células endoteliales de vena umbilical humana a redes de estructuras tubulares y promueve el crecimiento de vasos sanguíneos (16).

Visfatina (conocida también como factor estimulante de colonias de células pre-B o PBEF). Es una citocina que se expresa en el tejido graso visceral y 
sus niveles circulantes se correlacionan con la obesidad. La liberación de visfatina puede ser regulada por la glucosa y la insulina, e incrementa con la degradación progresiva de células beta (17). En mujeres gestantes, la concentración plasmática media de visfatina incrementa en el segundo trimestre de la gestación y disminuye en el último trimestre del embarazo (18). La visfatina está relacionada con el metabolismo de las HDL y existen reportes de su incremento en diabetes gestacional tipo 1 y tipo 2 y obesidad gestacional (17). Adicionalmente, se han encontrado incrementadas las concentraciones de visfatina séricas en el primer trimestre de la gestación en mujeres que desarrollaron preeclampsia por un mecanismo no relacionado a la perfusión placentaria alterada, y en mujeres que presentaron diabetes gestacional $(19,20)$.

\section{ESTRÉS OXIDATIVO}

El embarazo se acompaña de una demanda energética elevada, un incremento en los requerimientos de oxígeno y un aumento de la carga prooxidante, lo que normalmente produce un estado de estrés oxidativo. En la gestación normal, la tasa de producción de especies reactivas de oxígeno se compensa mediante su eliminación por factores antioxidantes enzimáticos y no enzimáticos (21); sin embargo, en embarazos complicados como preeclampsia, hipertensión asociada al embarazo y diabetes gestacional, la producción excesiva de especies reactivas de oxígeno vence a las defensas antioxidantes, lo cual conduce a un mayor grado de oxidación (21).

Las madres obesas tienen una baja actividad antioxidante total y bajas concentraciones de vitamina $C$ y $E$, actividades disminuidas de las enzimas glutatión peroxidasa y superóxido dismutasa; altos niveles de TG, radicales hidroperóxidos y grupos carbonilo en las proteínas comparado a las madres control con peso normal. De otro lado, los recién nacidos de madres obesas tienen disminuida tanto la actividad antioxidante total como las vitaminas antioxidantes; además tienen radicales hidroperóxidos incrementados comparado con los recién nacidos control. Por lo anterior, se ha sugerido que el imbalance oxidante/antioxidante es un factor importante causante de estrés fetal en las mujeres gestantes obesas (9).

Finalmente, se ha encontrado una asociación entre el IMC materno, los niveles de CT y la oxidación de las LDL en la madre, lo que puede contribuir al aumento de las complicaciones en el embarazo con obesidad (22). Los lípidos oxidados presentes en las LDL podrían causar la oxidación de membranas celulares endoteliales, ya que las LDL son tomadas por las células y los lípidos oxidados son incorporados en la membrana celular. La oxidación de membranas de células endoteliales permite que éstas pierdan proteínas y por lo tanto, podría explicar el edema y la proteinuria característicos de la preeclampsia (23).

\section{OMEGA 3 y 6}

Los ácidos grasos poli-insaturados (AGPI) omega-3, como el ácido eicosapentanoico (EPA) y omega-6, como el ácido araquidónico (AA) son componentes estructurales de las células del cuerpo e importantes para el desarrollo y el funcionamiento del sistema nervioso y visual de los humanos. Ambos ácidos grasos aportan fluidez a las membranas celulares, permitiendo el movimiento de proteínas en su superficie y dentro de la bicapa lipídica (24). Los ácidos grasos omega tienen varios efectos importantes para el organismo: son hipolipemiantes, antiinflamatorios o proinflamatorios y antioxidantes; el consumo de los ácidos grasos omega-3 y omega-6 durante el embarazo y la lactancia, beneficia el desarrollo del cerebro fetal e infantil y reduce los nacimientos prematuros (25). Durante la gestación, los ácidos grasos pasan de la madre al feto por transferencia placentaria; la placenta humana no tiene la capacidad para elongar y desaturar los AGPI precursores, sin embargo, es selectivamente permeable a los ácidos grasos de origen materno (1).

El efecto anti o proinflamatorio de los ácidos grasos omega-3 y omega-6, se lleva a cabo mediante la modulación de moléculas como la proteína $\mathrm{C}$ reactiva, la IL-6, el TNF y la leptina, los cuales son marcadores de inflamación e incrementan el número de receptores de insulina en varios tejidos (5). A partir de los ácidos grasos omega- 6 se derivan eicosanoides que modulan la respuesta inflamatoria, aumentando la vasodilatación y la permeabilidad vascular (24). Por su parte, los ácidos grasos omega-3, reducen la formación de los eicosanoides derivados de los ácidos grasos omega-6, y por este motivo, es conveniente mantener una dieta balanceada que contenga proporciones similares de ambos ácidos grasos, ya que comparten receptores en las mismas células diana por lo que sus efectos son antagónicos (24).

De otro lado, existen situaciones que pueden alterar el aporte nutricional materno-fetal, ya que pueden disminuir considerablemente las reservas de AGPI, entre ellas se destacan: la alimentación inadecuada, el consumo de grasas y aceites con alta proporción de omega-6 y bajo aporte de omega-3; embarazos periódicos en los que la madre no alcanza a recuperar las reservas de ácidos grasos para la siguiente gestación o un embarazo múltiple (26). Se estima que el feto durante el último tercio del período gestacional, y el recién nacido durante los primeros 6 meses de vida, requieren de un aporte significativo de AA y de EPA, debido a que la velocidad de transformación de sus precursores hepáticos no es suficiente para cubrir los requerimientos metabólicos 
de estos ácidos grasos. De esta forma, se estima que la dieta materna influye en la fisiología del crecimiento prenatal y posnatal; la nutrición inadecuada del embrión afecta la vida después del nacimiento, y los efectos de la dieta materna en la gestación temprana son especialmente importantes (26).

A continuación se describen algunos efectos benéficos que tiene el consumo de ácidos grasos omega-3 en la gestación, los cuales han sido ampliamente estudiados.

Efecto hipolipemiante. Se produce a través de la actividad de dos factores de transcripción que interactúan con los ácidos grasos de cadena larga: 1) el receptor activado por el proliferador de peroxisomas, que participa en la activación de los procesos de oxidación de los ácidos grasos, y 2) la proteína fijadora del elemento regulador del CT, que participa en la inhibición de la síntesis de TG. La interacción entre estos factores de transcripción permite inhibir la síntesis de ácidos grasos reduciendo la disponibilidad del sustrato para la producción de TG (27).

Regulación de la actividad del factor nuclear kappa $B$ (NF- kB). NF- kB es generalmente activado por el TNF; posee un papel bifásico en el proceso inflamatorio local y puede inducir la expresión de moléculas de adhesión, como también disminuir las alteraciones de la función endotelial al reducir la producción de óxido nítrico (28).

Efectos generales sobre el embarazo y el neonato. Los ácidos grasos omega-3 tienen un moderado efecto sobre la mayor duración del embarazo y el peso al nacer pero no son suficientes para plantear una recomendación general con el fin de evitar los partos prematuros. De otro lado, la mayoría de los trabajos de investigación han encontrado diferencias estadísticamente significativas bien sea en pruebas visuales o cognitivas en la descendencia y el efecto de los omega-3 en la disminución de biomarcadores alergénicos en los infantes; además, se ha observado que la suplementación de estos ácidos grasos puede que no tenga un beneficio medible, pero su bajo consumo puede tener repercusiones graves principalmente en bebés nacidos prematuramente. Finalmente, se ha descrito que tanto el exceso como el déficit de ácidos grasos omega-3 en la dieta pueden causar retraso del crecimiento postnatal, y alteraciones sensoriales y neurológicas en la descendencia (29).

\section{CONCLUSIONES}

Durante un embarazo normal cambian la mayoría de los componentes del perfil lipídico, aumenta el estrés oxidativo y persiste una respuesta inflamatoria moderada, pero en un embarazo con obesidad, hay un aumento exacerbado de estos mecanismos, aumentando la morbimortalidad materna y fetal, como un factor determinante para las enfermedades pre y postparto que pueden sufrir ambos. Por su parte, es importante resaltar que el equilibrio en cuanto a los requerimientos de ácidos grasos omega-3 y omega-6, puede contribuir con la regulación de los componentes del perfil lipídico alterados; de igual manera, la capacidad antioxidante y antiinflamatoria de los ácidos grasos omega-3, puede contrarrestar algunos efectos adversos de la obesidad en el embarazo.

FUENTES DE FINANCIACIÓN. Este trabajo fue financiado por Sostenibilidad Universidad de Antioquia 2013-2014 y Fondo Doctorados Nacionales- COLCIENCIAS.

\section{REFERENCIAS}

1. Herrera E. Metabolic adaptations in pregnancy and their implications for the availability of substrates to the fetus. Eur J Clin Nutr 2000;54 Suppl 1:S47-51.

2. Wiznitzer A, Mayer A, Novack V, Sheiner E, Gilutz H, Malhotra A, et al. Association of lipid levels during gestation with preeclampsia and gestational diabetes meIlitus: a population-based study. Am J Obstet Gynecol 2009;201(5):482 e1-8.

3. Mardones F, Rosso P. [Design of a weight gain chart for pregnant women]. Rev Med Chil 1997;125(12):143748.

4. Sirimi N, Goulis DG. Obesity in pregnancy. Hormones (Athens) 2010;9(4):299-306.

5. Rodríguez M, Tovar A, Del Prado M, Torres N. Mecanismos moleculares de acción de los ácidos grasos poliinsaturados y sus beneficios en la salud. Rev Invest Clin 2005;57(3):457-72.

6. Lithell $\mathrm{H}$. Hypertension and hyperlipidemia. A review. Am J Hypertens 1993;6(11 Pt 2):303S-8S.

7. Abduljalil K, Furness P, Johnson TN, Rostami-Hodjegan A, Soltani H. Anatomical, physiological and metabolic changes with gestational age during normal pregnancy: a database for parameters required in physiologically based pharmacokinetic modelling. Clin Pharmacokinet 2012;51(6):365-96.

8. Belo L, Caslake M, Gaffney D, Santos-Silva A, Pereira-Leite L, Quintanilha A, et al. Changes in LDL size and HDL concentration in normal and preeclamptic pregnancies. Atherosclerosis 2002;162(2):425-32.

9. Ray JG, Vermeulen MJ, Shapiro JL, Kenshole AB. Maternal and neonatal outcomes in pregestational and gestational diabetes mellitus, and the influence of maternal obesity and weight gain: the DEPOSIT study. Diabetes Endocrine Pregnancy Outcome Study in Toronto. QJM 2001;94(7):347-56.

10. Adamczak M, Wiecek $A$. The adipose tissue as an endocrine organ. Semin Nephrol 2013;33(1):2-13.

11. D'Ippolito S, Tersigni C, Scambia G, Di Simone N. Adipokines, an adipose tissue and placental product with biological functions during pregnancy. Biofactors 2012;38(1):14-23.

12. Di Simone N, Di Nicuolo F, Sanguinetti M, Castellani R, D'Asta M, Caforio L, et al. Resistin regulates 
human choriocarcinoma cell invasive behaviour and endothelial cell angiogenic processes. J Endocrinol 2006;189(3):691-9.

13. Yura S, Sagawa N, Itoh H, Kakui K, Nuamah MA, Korita $D$, et al. Resistin is expressed in the human placenta. J Clin Endocr Metab 2003;88(3):1394-7.

14. Hendler I, Blackwell SC, Mehta SH, Whitty JE, Russell $\mathrm{E}$, Sorokin $\mathrm{Y}$, et al. The levels of leptin, adiponectin, and resistin in normal weight, overweight, and obese pregnant women with and without preeclampsia. Am J Obstet Gynecol 2005;193(3 Pt 2):979-83.

15. McDonald EA, Wolfe MW. The pro-inflammatory role of adiponectin at the maternal-fetal interface. Am J Reprod Immunol 2011;66(2):128-36.

16. Ouchi N, Kobayashi H, Kihara S, Kumada M, Sato K, Inoue $\mathrm{T}$, et al. Adiponectin stimulates angiogenesis by promoting cross-talk between AMP-activated protein kinase and Akt signaling in endothelial cells. J Biol Chem 2004;279(2):1304-9.

17. Mastorakos G, Valsamakis G, Papatheodorou DC, Barlas I, Margeli A, Boutsiadis A, et al. The role of adipocytokines in insulin resistance in normal pregnancy: visfatin concentrations in early pregnancy predict insulin sensitivity. Clin Chem 2007;53(8):1477-83.

18. Mazaki-Tovi S, Romero R, Kusanovic JP, Vaisbuch E, Erez O, Than NG, et al. Maternal visfatin concentration in normal pregnancy. J Perinat Med 2009;37(3):206-17.

19. Ferreira AF, Rezende JC, Vaikousi E, Akolekar R, Nicolaides $\mathrm{KH}$. Maternal serum visfatin at 11-13 weeks of gestation in gestational diabetes mellitus. Clin Chem 2011;57(4):609-13.

20. Ferreira AF, Rezende JC, de Cassia COR, Akolekar $\mathrm{R}$, Nicolaides $\mathrm{KH}$. Maternal serum visfatin at 11-13 weeks' gestation in preeclampsia. J Hum Hypertens 2013;27(4):261-4.
21. Sharma JB, Sharma A, Bahadur A, Vimala N, Satyam A, Mittal S. Oxidative stress markers and antioxidant levels in normal pregnancy and pre-eclampsia. Int $\mathrm{J}$ Gynaecol Obstet 2006;94(1):23-7.

22. Gutierrez A. Estrés oxidativo en la gestación: ¿una nueva óptica en la atención a la embarazada? Rev Cubana Obstet Ginecol 2005;31.

23. Vanderlelie J, Venardos K, Clifton VL, Gude NM, Clarke FM, Perkins AV. Increased biological oxidation and reduced anti-oxidant enzyme activity in pre-eclamptic placentae. Placenta 2005;26(1):53-8.

24. Kalish BT, Fallon EM, Puder M. A tutorial on fatty acid biology. JPEN J Parenter Enteral Nutr 2012;36(4):3808.

25. Greenberg JA, Bell SJ, Ausdal WV. Omega-3 Fatty Acid supplementation during pregnancy. Rev Obstet Gynecol 2008;1(4):162-9.

26. Valenzuela A, Nieto S. Ácidos grasos omega-6 y omega-3 en la nutrición perinatal: su importancia en el desarrollo del sistema nervioso y visual. Rev Chil Pediatr 2003;2(74):149-57.

27. Bourre JM. Dietary omega-3 fatty acids for women. Biomed Pharmacother 2007;61(2-3):105-12.

28. Martinez-Micaelo N, Gonzalez-Abuin N, Terra X, Richart C, Ardevol A, Pinent M, et al. Omega-3 docosahexaenoic acid and procyanidins inhibit cyclo-oxygenase activity and attenuate NF-kappaB activation through a p105/p50 regulatory mechanism in macrophage inflammation. Biochem J 2012;441(2):653-63.

29. Larque E, Gil-Sanchez A, Prieto-Sanchez MT, Koletzko B. Omega 3 fatty acids, gestation and pregnancy outcomes. Br J Nutr 2012;107 Suppl 2:S77-84. 